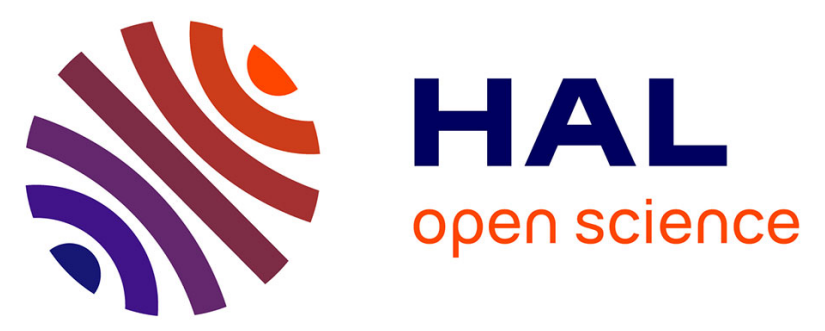

\title{
Short-term stability of Cs microcell-stabilized lasers using dual-frequency sub-Doppler spectroscopy
}

Anthony Gusching, Michael Petersen, Nicolas Passilly, Denis Brazhnikov, Moustafa Abdel Hafiz, Rodolphe Boudot

\section{To cite this version:}

Anthony Gusching, Michael Petersen, Nicolas Passilly, Denis Brazhnikov, Moustafa Abdel Hafiz, et al.. Short-term stability of Cs microcell-stabilized lasers using dual-frequency sub-Doppler spectroscopy. Journal of the Optical Society of America B, 2021, 38 (11), pp.3254 - 3260. 10.1364/JOSAB.438111 . hal-03403179

\author{
HAL Id: hal-03403179 \\ https://hal.science/hal-03403179
}

Submitted on 26 Oct 2021

HAL is a multi-disciplinary open access archive for the deposit and dissemination of scientific research documents, whether they are published or not. The documents may come from teaching and research institutions in France or abroad, or from public or private research centers.
L'archive ouverte pluridisciplinaire HAL, est destinée au dépôt et à la diffusion de documents scientifiques de niveau recherche, publiés ou non, émanant des établissements d'enseignement et de recherche français ou étrangers, des laboratoires publics ou privés. 


\title{
Short-term stability of Cs microcell-stabilized lasers using dual-frequency sub-Doppler spectroscopy
}

\author{
BOUDOT $^{1}$ \\ ${ }^{1}$ FEMTO-ST, CNRS, Université Bourgogne Franche-Comté, 26 rue de l'épitaphe 25030 Besançon, France \\ ${ }^{2}$ Novosibirsk State University, 1 Pirogov Street, 630090 Novosibirsk, Russia \\ ${ }^{3}$ Institute of Laser Physics SB RAS, 15B Lavrentiev Avenue, 630090 Novosibirsk, Russia \\ *Corresponding author: rodolphe.boudot@femto-st.fr
}

A. Gusching ${ }^{1}$, M. Petersen ${ }^{1}$, N. Passilly ${ }^{1}$, D. Brazhnikov 2,3 , M. Abdel Hafiz ${ }^{1}$, and R.

Compiled October 5, 2021

The combination of atomic spectroscopy, integrated photonics and microelectromechanical systems (MEMS) paves the road to the demonstration of microcell-based optical atomic clocks. Here, we report the short-term stability budget of table-top Cs microcell-stabilized lasers based on dual-frequency sub-Doppler spectroscopy (DFSDS). The dependence of the sub-Doppler resonance properties on key experimental parameters is studied. The detection noise budget and absolute phase noise measurements are in good agreement with the measured short-term frequency stability of the laser beatnote, at the level of $1.1 \times 10^{-12} \tau^{-1 / 2}$ until $100 \mathrm{~s}$, currently limited by the intermodulation effect from a distributed-feedback laser setup. The fractional frequency stability of the laser beatnote at $1 \mathrm{~s}$ is about 100 times better than those of commercial microwave chip-scale atomic clocks and validate the interest of the DFSDS approach for the development of high-performance microcell-based optical standards. () 2021 Optical Society of America

http://dx.doi.org/10.1364/ao.XX.XXXXXX

\section{INTRODUCTION}

The interrogation of an alkali vapor atom ensemble in a mmscale cell has allowed the development of a wide variety of high-precision chip-scale atomic devices [1]. Among these instruments, miniaturized microwave atomic clocks based on coherent population trapping (CPT) [2] have known a remarkable development and progress, including their commercialization [3], due to their unrivaled size-power-frequency stability budget [4-7]. These clocks are now widely-used in numerous applications, including underwater sensor networks, secure communications or satellite-based navigation systems.

However, microwave CSACs suffer from drawbacks and limitations. These clocks use in general vertical-cavity surfaceemitting lasers (VCSELs) whose frequency noise and variations can limit the clock frequency stability $[7,8]$. In addition, the presence of buffer gas pressure in the cell induces an important frequency shift of the clock transition [9] that can limit the clock mid-term frequency stability and jeopardize the establishment of a clock accuracy budget.

Over the last decade, state-of-the-art microwave atomic clocks (i.e. fountains [10]) have been outperformed by optical atomic clocks that rely on the frequency stabilization of a low noise laser onto a high quality-factor optical atomic transition [1116]. However, these instruments, making use of laser cooling, lattice-confined atoms or trapped ions, remain complex and their deployment outside the laboratory is challenging. To satisfy applications with stringent SWaP (size-weight-power) specifications, an attractive approach concerns the development of high-performance vapor cell-based optical clocks using Dopplerfree interrogation schemes [17].

In this domain, the two-photon transition at $778 \mathrm{~nm}$ in $\mathrm{Rb}$ vapor is an attractive candidate due to its narrow natural linewidth of about $300 \mathrm{kHz}$ [18-20]. This approach was recently used for the demonstration of a microcell-based optical clock with remarkable stability performances at the level of $4 \times 10^{-12} \tau^{-1 / 2}$ until $1000 \mathrm{~s}$ [21], later improved to $2.9 \times 10^{-12} \tau^{-1 / 2}$ until 100 $\mathrm{s}$ using a micro-optics breadboard [22]. More recently, using a low noise external cavity diode laser (ECDL), the same group at NIST reported a Rb-microcell optical frequency standard with an exceptional frequency stability of $1.8 \times 10^{-13} \tau^{-1 / 2}$ until $100 \mathrm{~s}$ [23]. The development of high-performance cell optical clocks based on the alternative 780-776 nm two-photon transition in $\mathrm{Rb}$ was also reported [24, 25] but, to our knowledge, no demonstration of this approach has been performed with a MEMS cell yet. An alternative approach to the two-photon transition is the well-known sub-Doppler spectroscopy (SDS) scheme [26, 27]. The latter has been widely-used for laser frequency stabilization with glass-blown vapor cells [28-33]. The use of SDS for the development of microcell-based frequency-stabilized lasers was investigated initially in [34] with the demonstration of a micro-fabricated saturated absorption laser spectrometer and in further studies [35-37]. In [37], the frequency stabilization of a $\mathrm{DBR}$ laser onto a $\mathrm{Rb}$ microcell, using some light routing through an integrated silicon nitride waveguide and grating system to the cell, was demonstrated at the level of $10^{-11}$ for time scales up to $10^{4} \mathrm{~s}$. SDS was also explored in hybrid setups, in combination 

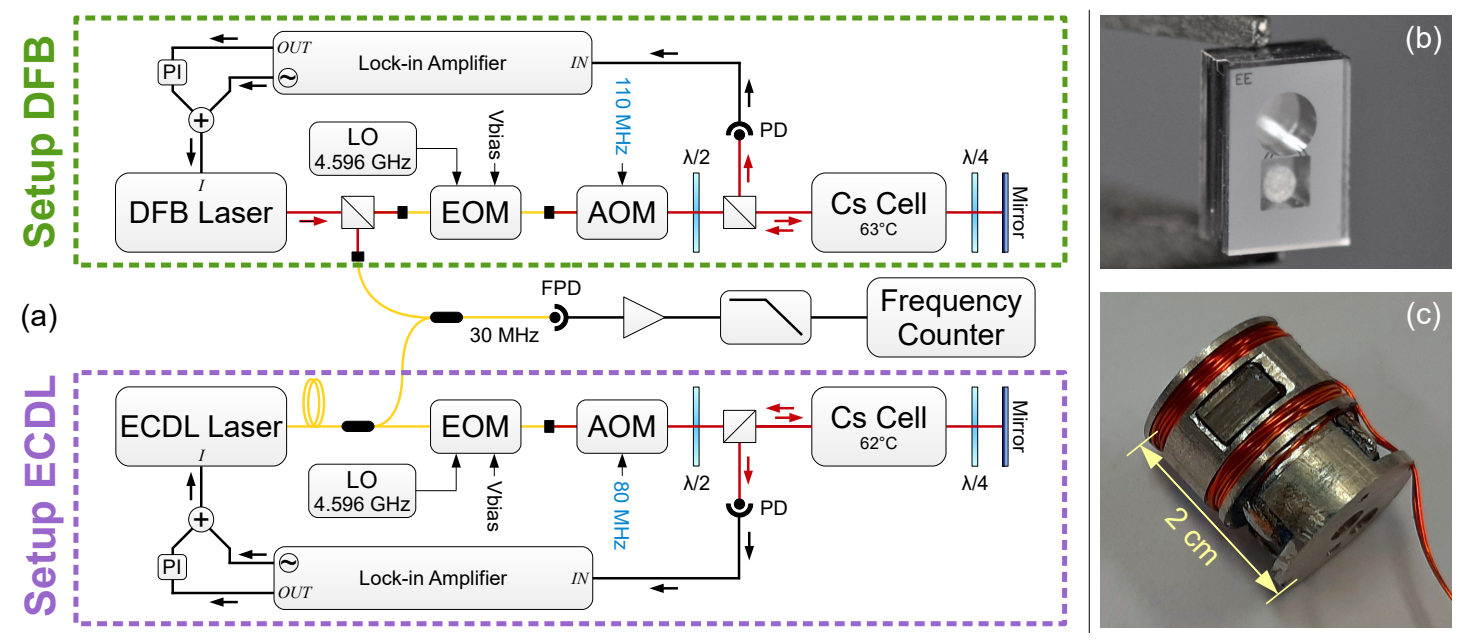

Fig. 1. (a) Schematic of the experimental setup made of two microcell-stabilized lasers using dual-frequency sub-Doppler spectroscopy (DFSDS). Yellow lines indicate fibered sections. See the text for description. (b) Photograph of a Cs microfabricated cell. (c) Photograph of the microcell physics package.

with a microresonator comb for direct-comb spectroscopy [38] or an ultra-compact Fabry-Perot cavity [39].

In $[40,41]$, the detection of high-contrast sign-reversed naturallinewidth sub-Doppler resonances, explained in a detailed quantitative model [42], was reported using a dual-frequency subDoppler spectroscopy (DFSDS) technique. However, no detailed resonance spectroscopy and short-term stability budget were performed.

In the present paper, we report a detailed short-term stability budget of table-top Cs microcell-stabilized lasers based on DFSDS. Two similar systems inside the same thermal enclosure are compared. The first setup uses a distributed feedback (DFB) laser while the second setup uses an external-cavity diode laser (ECDL). Spectroscopy of the sub-Doppler resonance as a function of some key experimental parameters (laser power, cell temperature and magnetic field) was performed first in order to optimize the lasers' short-term frequency stability. A detailed noise analysis is then reported for each setup and found to be in good agreement with the measured short-term stability of $1.1 \times 10^{-12} \tau^{-1 / 2}$ up to $100 \mathrm{~s}$ and absolute phase noise performances.

\section{EXPERIMENTAL SETUP}

Figure 1 shows the experimental setup. Two lasers (one distributed feedback (DFB, Eagleyard EYP-DFB-0895 model) laser and one external cavity diode laser (ECDL, Toptica DL Pro) are each frequency-stabilized onto a Cs microcell using DFSDS. The first laser system uses a DFB laser diode tuned on the Cs $D_{1}$ line at $895 \mathrm{~nm}$ followed by a $70 \mathrm{~dB}$ optical isolation stage. The light is fiber-coupled into a Mach-Zehnder electro-optic modulator (EOM, iXblue NIR-MX800-LN-10) driven by a microwave frequency synthesizer at $4.596 \mathrm{GHz}$ in order to produce two first-order optical sidebands split by $9.192 \mathrm{GHz}$. The light is then sent into an acousto-optic modulator (AOM) driven at 110 $\mathrm{MHz}$ by a RF synthesizer. The AOM can be used for laser power control and stabilization [43]. The +1 -order is then directed into an evacuated Cs vapor microfabricated cell containing a pill Cs dispenser [44]. The laser beam diameter at the cell input is about $2 \mathrm{~mm}$. The cell is made of silicon cavities etched by deep reactive ion etching (DRIE) and sandwiched between two anodically-bonded borosilicate glass wafers. During the cell fabrication, laser-activation of the pill dispenser is performed gradually, i.e. with successive steps, in order to avoid excess of alkali in the cell. Figure 1(b) shows a photograph of the microfabricated cell. Note that, over a period of several months, at a cell temperature of $62^{\circ} \mathrm{C}$ used in clock operation, we did not observe any significant appearance of Cs droplets on the cell windows. The MEMS cell is held inside the physics package shown in Fig. 1(c), which is surrounded by a mu-metal magnetic shield. After the cell, the light impinges a quarter-wave plate (QWP) and is reflected back by a mirror positioned to enhance the constructive contribution of hyperfine Zeeman effects and increase the sub-Doppler resonance contrast [42]. The reflected light beam is then directed using a cube right before the cell to a photodiode for the detection of the spectroscopic signal and the generation of a derivative error signal using a lock-in amplifier (Stanford Research SR-860). The latter is then processed in a proportional-integral (PI) controller to provide a correction signal that is applied to the DFB laser current.

The second laser system is based on an ECDL. Similar EOM and AOM references are used, except that the AOM of the ECDL setup is driven at $80 \mathrm{MHz}$ (instead of $110 \mathrm{MHz}$ for the DFB setup). This allows to directly produce a $30 \mathrm{MHz}$ beat-note between the two laser setups. Before the EOM, on both setups, a part of the laser beam is separated from the main beam and fiber coupled. The two laser beams are then combined into one single fiber and the resulting beat-note signal is measured by a fast photo-diode. The output signal can be analyzed with a frequency counter or a phase noise analyzer. The counter and both microwave frequency synthesizers of the experiment are all referenced to an active hydrogen maser.

\section{EXPERIMENTAL RESULTS}

\section{A. Spectroscopy of the sub-Doppler resonance}

Figure 2 shows spectra of sub-Doppler resonances, detected in the bottom of absorption profiles, for both the DFB and ECDL setups, used in clock configuration. The total laser power at the input of the cell is about $450 \mu \mathrm{W}$. Due to a more favorable signal, our study was performed by locking the laser frequency to the Cs atom $F^{\prime}=4$ excited state. In this test, the signal height $S$ of 

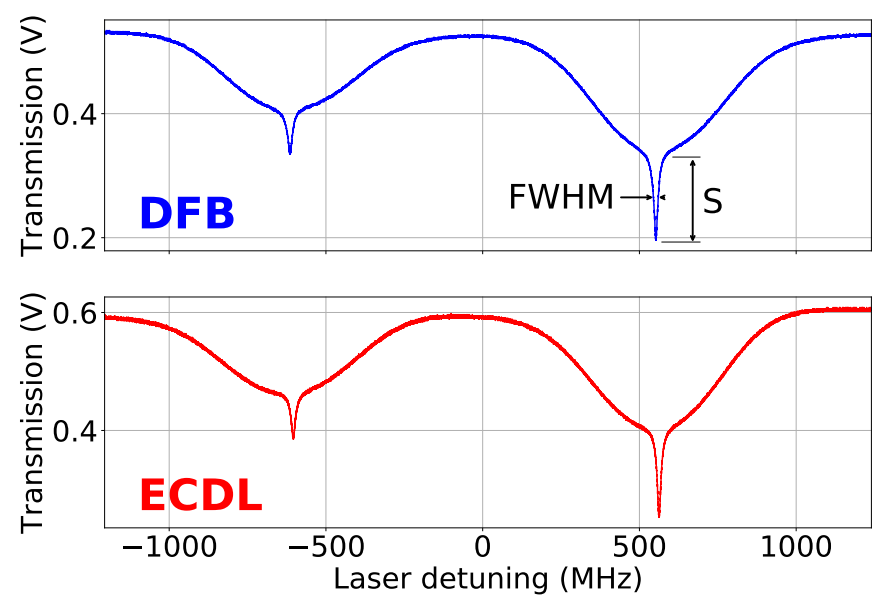

Fig. 2. Spectra of sub-Doppler resonances detected in Cs vapor microfabricated cells, for optimized clock short-term stability, for both laser setups. Cell temperatures are $63^{\circ} \mathrm{C}$ and $62^{\circ} \mathrm{C}$, for the DFB and ECDL setup respectively.
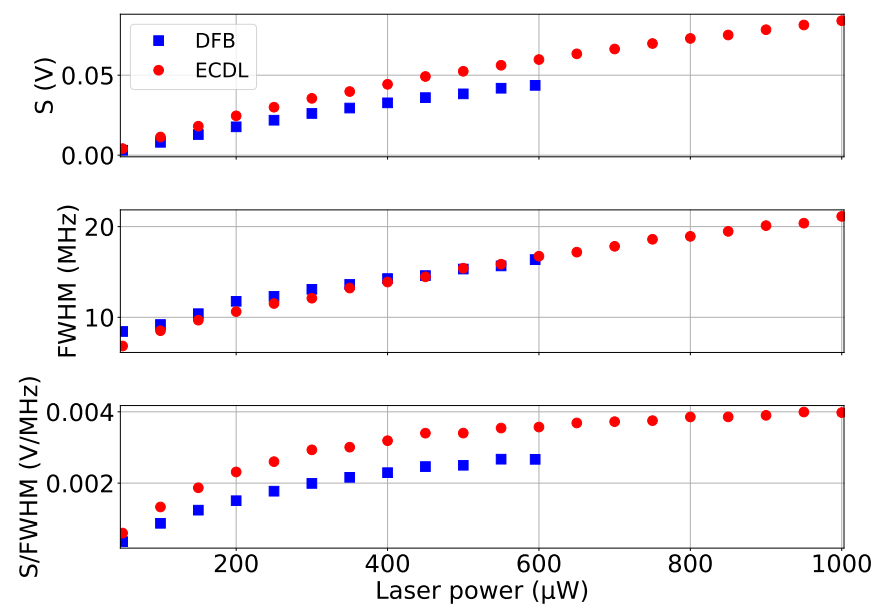

Fig. 3. Signal height, FWHM and signal/FWHM ratio of the sub-Doppler resonance versus the laser power entering the MEMS cell, for both laser setups. The cell temperatures are 63 and $62^{\circ} \mathrm{C}$, for the DFB and ECDL setups respectively.

the sub-Doppler resonance is $126 \mathrm{mV}$ for the DFB setup and 138 $\mathrm{mV}$ for the ECDL setup. The FWHM of the sub-Doppler resonance is $14.7 \mathrm{MHz}$ and $14.2 \mathrm{MHz}$ for the DFB and ECDL setup, respectively. Spectroscopy of these sub-Doppler resonances was performed to evaluate the impact of key experimental parameters, mainly the laser power, the cell temperature and the static magnetic field.

Figure 3 depicts the signal height $S$, the FWHM and the $S$ /FWHM ratio versus the laser power measured right in front of the cell for both setups. In the DFB setup case, no data points were recorded for laser power higher than $600 \mu \mathrm{W}$, due to limited power at the output of the AOM. We observe a clear increase of the resonance signal and FWHM with increased laser power. The slope of the S/FWHM ratio dependence to laser power is reduced for power values higher than about $300 \mu \mathrm{W}$.

We did a similar study with the cell temperature, with results shown in Fig. 4. In both setups, the signal is maximized at a temperature of about $65-67^{\circ} \mathrm{C}$, while the FWHM reaches a
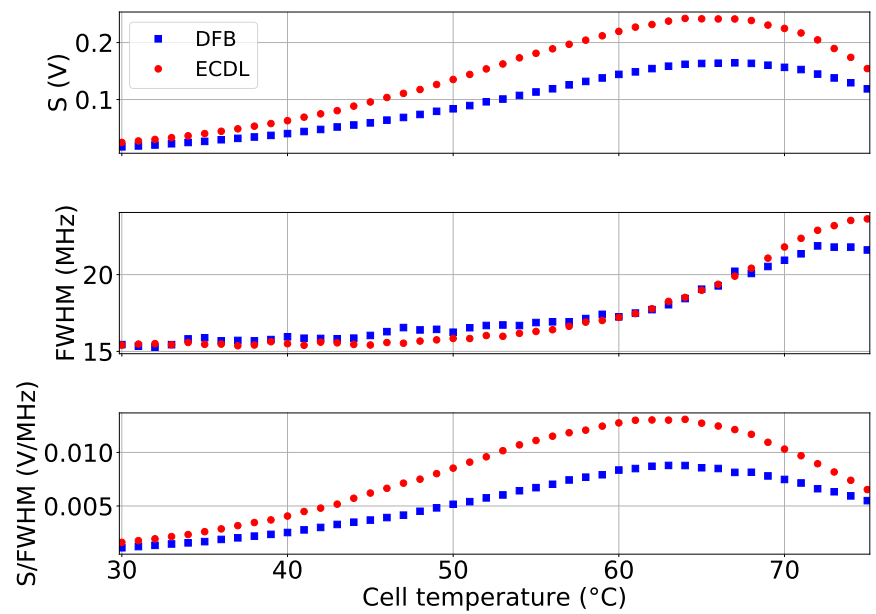

Fig. 4. Signal height, FWHM and signal/FWHM ratio of the sub-Doppler resonance versus the cell temperature incident in the MEMS cell, for both laser setups. For this measurement, the laser power is $600 \mu \mathrm{W}$ for both the DFB and ECDL setups.

maximum above $72^{\circ} \mathrm{C}$. The trend of the S/FWHM ratio curve shows a maximum at $63^{\circ} \mathrm{C}$ and $62^{\circ} \mathrm{C}$ for DFB and ECDL setups, respectively. We note that the ECDL setup yields a S/FWHM ratio about $30 \%$ higher than the DFB setup.

A last investigation was performed on the static magnetic field
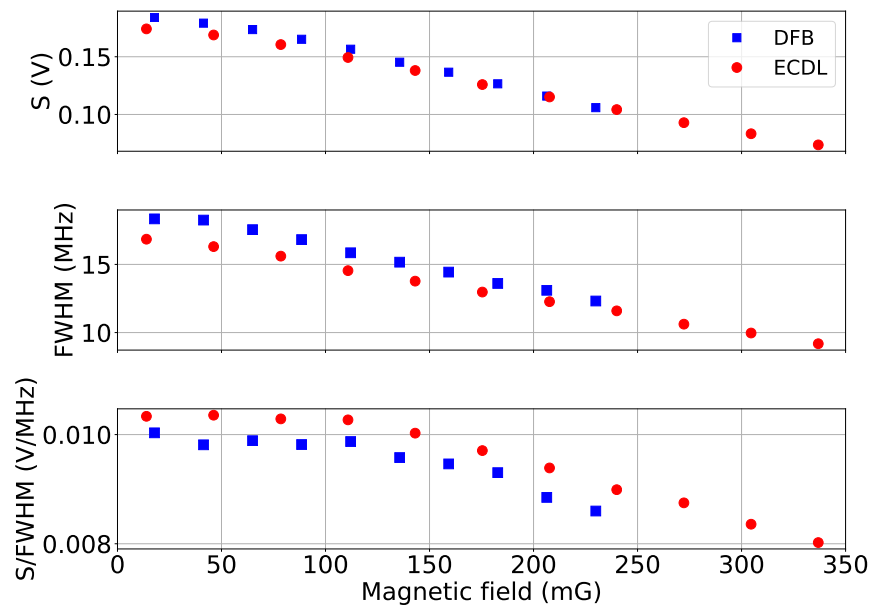

Fig. 5. FWHM, signal and signal/FWHM ratio of the subDoppler resonance versus the static magnetic field experienced by the atoms, for both laser setups. The cell temperatures are 63 and $62^{\circ} \mathrm{C}$, for the DFB and ECDL setups respectively.

$B$ experienced by the atoms by changing the current applied to the Helmholtz coils (see Fig. 1(c)). Calibration of the magnetic field-versus-current dependence was performed by replacing the Cs cell by a buffer-gas filled Cs-Ne microcell, and the reflexion mirror by a photodiode to detect laser power at the direct output of the cell and scanning the microwave frequency to detect microwave CPT resonances. The application of the static magnetic field raises the Zeeman degeneracy, from which the actual applied magnetic field was extracted by measuring the frequency change of the magnetic-field sensitive hyperfine Zeeman transition between both $m_{F}=+1$ sub-levels (with $m_{F}$ the 

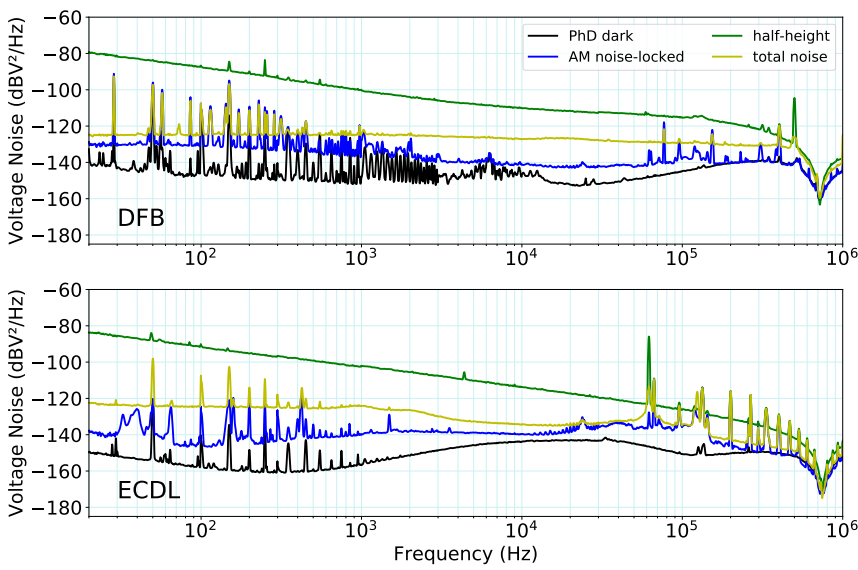

Fig. 6. Detection noise at the photodiode output for both setups (DFB and ECDL). Measurements are performed with a phase noise analyzer (Rohde-Schwarz FSWP). Black curves represent the detection noise with the photodiode in the dark. Blue curves depict an image of the laser amplitude (AM) noise, with activation of the laser power servo. For this purpose, the laser frequency is tuned out of optical resonance and the laser power impinging the photodiode is reduced with a neutral density filter to be comparable to the incident one in clock configuration. Green curves yield an image of the free-running laser frequency noise (FM). Light green curves correspond to the total detection noise at the photodiode output in locked laser frequency configuration.

quantum number) with the applied current. The sub-Doppler resonance is narrowed with increased magnetic field. This narrowing effect, predicted in [41] (fig. 20), is explained by the increase of the Doppler profile height with higher values of $B$, in conjunction with the fact that, under low magnetic field values, the absorption value at the center of the resonance does not change. Note also that the signal/FWHM ratio of sub-Doppler resonances is maximized as expected at null magnetic field for both setups [41].

\section{B. Noise budget and absolute phase noise}

Main noise source contributions to the short-term frequency stability of each laser setup are the photon shot noise, the photodetector noise (in the dark), the laser amplitude (AM) noise, the laser frequency noise through the FM-AM conversion process and the intermodulation effect due to the intrinsic phase noise (frequency noise) of the free-running laser. For a first estimation, we only consider white-noise sources.

The shot-noise contribution $\sigma_{s n}$ is given by [45]:

$$
\sigma_{s n}(\tau)=\sqrt{\frac{\Delta v^{2}}{v_{0}} \frac{2 h v_{0}}{C^{2} P_{o}} \frac{1}{\tau}}
$$

with $\Delta v$ being the sub-Doppler resonance linewidth (FWHM), $h$ the Planck constant, $v_{0}$ the laser frequency $\left(3.35 \times 10^{14} \mathrm{~Hz}\right.$ for the Cs $\mathrm{D}_{1}$ line), $P_{o}$ the optical power incident on the photodiode and $C$ the contrast of the resonance, defined as the ratio between the sub-Doppler resonance signal height $S$ and the dc background level in the bottom of the sub-Doppler resonance.

The contribution of the photodetector noise $\sigma_{p d}$ is given by:

$$
\sigma_{p d}(\tau)=\sqrt{\frac{1}{v_{0}^{2}} \frac{\sigma_{D}^{2}}{S_{l}^{2}} \frac{1}{\tau}}
$$

Table 1. Short-term noise and stability budget, for both DFB and ECDL setups.

\begin{tabular}{lll}
\hline Properties & DFB & ECDL \\
\hline$S(\mathrm{mV})$ & 126 & 139 \\
FWHM $(\mathrm{MHz})$ & 14.7 & 14.2 \\
$S_{l}(\mathrm{~V} / \mathrm{Hz})$ & $8.6 \times 10^{-9}$ & $9.8 \times 10^{-9}$ \\
$C$ & 0.65 & 0.55 \\
Absorption $(\%)$ & 63.1 & 58.6 \\
$P_{i}(\mu \mathrm{W})$ & 450 & 450 \\
$P_{o}(\mu \mathrm{W})$ & 166 & 186.5 \\
$f_{M}(\mathrm{kHz})$ & 500 & 61.95 \\
\hline Noise source & $\mathrm{DFB}$ & $\mathrm{ECDL}$ \\
\hline$\sigma_{s n}(1 \mathrm{~s})$ & $3.5 \times 10^{-15}$ & $3.8 \times 10^{-15}$ \\
$\sigma_{p d}(1 \mathrm{~s})$ & $3.9 \times 10^{-14}$ & $6 \times 10^{-14}$ \\
$\sigma_{A M-A M}(1 \mathrm{~s})$ & $3.8 \times 10^{-14}$ & $3.0 \times 10^{-13}$ \\
$\sigma_{F M-A M}(1 \mathrm{~s})$ & $4.4 \times 10^{-13}$ & $4.3 \times 10^{-13}$ \\
$\sigma_{i n t}(1 \mathrm{~s})$ & $1.5 \times 10^{-12}$ & $5.75 \times 10^{-13}$ \\
\hline$\sigma_{y}(1 \mathrm{~s})$ & $1.6 \times 10^{-12}$ & $7.8 \times 10^{-13}$ \\
\hline
\end{tabular}

with $\sigma_{D}^{2}$ being the power spectral density (PSD) of voltage noise at the output of the photodiode in the dark measured at the offset frequency $f=f_{M}$, where $f_{M}$ is the laser modulation frequency, while $S_{l}=S / \Delta v$ is the sub-Doppler resonance signal/linewidth ratio. The contribution of laser AM and laser FM noise is extracted in a similar way by measuring the PSD of voltage noise at $f_{M}$ in respective conditions where the laser frequency is out of optical resonance or at half-height of the sub-Doppler resonance profile, respectively.

The free-running laser phase noise can limit the final laser shortterm frequency stability through the intermodulation effect [46], with the contribution:

$$
\sigma_{i n t}(\tau) \simeq \frac{f_{M}}{v_{0}} \sqrt{S_{\varphi}\left(2 f_{M}\right)} \sqrt{\frac{1}{\tau}}
$$

with $S_{\varphi}\left(2 f_{M}\right)$ the PSD of the laser phase noise fluctuations in $\operatorname{rad}^{2} / \mathrm{Hz}$ measured at $f=2 f_{M}$.

The detection noise can be also degraded by the laser amplitude (AM) noise and the intrinsic laser frequency noise (FM). Their contributions, $\sigma_{A M-A M}$ and $\sigma_{F M-A M}$, are respectively obtained by:

$$
\begin{gathered}
\sigma_{A M-A M}(\tau) \simeq \sqrt{\left(\frac{1}{v_{0}}\right)^{2} \frac{\sigma_{P}^{2}(\Delta V / \Delta P)^{2}}{S_{l}^{2}} \frac{1}{\tau}} \\
\sigma_{F M-A M}(\tau) \simeq \sqrt{\left(\frac{1}{v_{0}}\right)^{2} \frac{\sigma_{v_{0}}^{2}\left(\Delta V / \Delta v_{0}\right)^{2}}{S_{l}^{2}}} \frac{1}{\tau}
\end{gathered}
$$

with $\sigma_{P}^{2}$ the PSD of laser power fluctuations, in $\mathrm{W}^{2} / \mathrm{Hz}$, at $f=f_{M}$ (measured without the cell for a laser power $P_{o}$ impacting the photodiode) and $\Delta V / \Delta P$ is the fluctuation of the clock resonance signal induced by a laser power variation in 


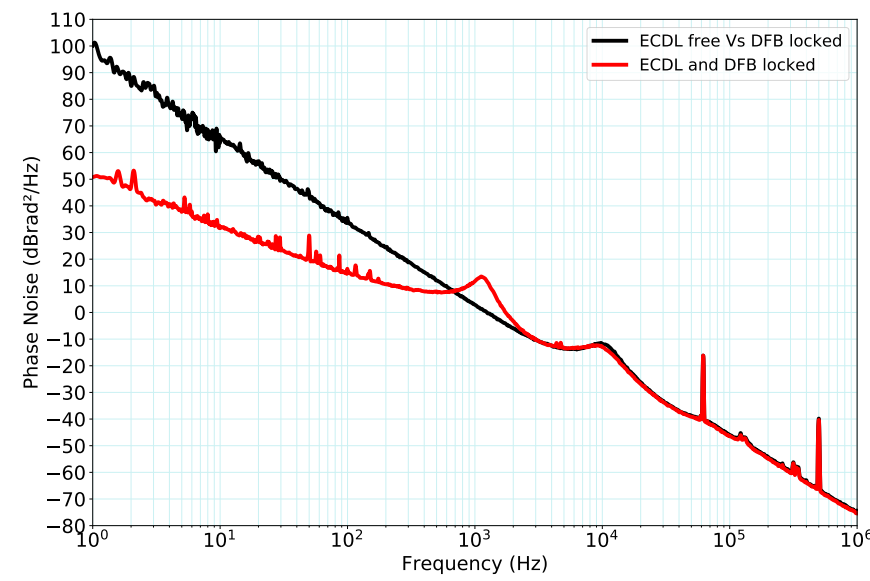

Fig. 7. Absolute phase noise of the laser beatnote in different configurations: free-running ECDL vs locked DFB, or both locked.

$\mathrm{V} / \mathrm{W}$. We note also $\sigma_{v_{0}}^{2}$ and $\Delta V / \Delta v_{0}$ the measured signal sensitivity to the laser frequency in $\mathrm{V} / \mathrm{Hz}$.

Following the spectroscopy tests described above, we performed a detection noise analysis on both setups in order to evaluate the contribution of main noise sources on the laser beatnote short-term frequency stability. Corresponding noise spectra are shown, for both setups, in Fig. 6. Using equations reported above and resonance spectra shown in Fig. 2, Table 1 summarizes the key parameters of each laser setup and the respective contributions to the short-term noise budget. We calculate that the laser beatnote short-term stability should be limited at the level of about $1.6 \times 10^{-12}$ at $1 \mathrm{~s}$ by the DFB setup. In the DFB setup, the main noise contribution to the total budget is the laser phase noise through the intermodulation effect. The expected short-term stability at $1 \mathrm{~s}$ of the ECDL setup is estimated at the level of $7.8 \times 10^{-13}$ at $1 \mathrm{~s}$ and is currently mainly limited by the intermodulation effect and the FM-AM noise conversion process. We note that the contribution of the total detection noise of the ECDL setup has the potential to be reduced by about $10 \mathrm{~dB}$ by operating the ECDL setup with $f_{m}=500 \mathrm{kHz}$ instead of $62 \mathrm{kHz}$ ( $f_{m}$ currently limited by the ECDL head controller electronics). In this case, the short-term instability of the ECDL setup should be reduced to a few $\times 10^{-13}$ at $1 \mathrm{~s}$.

For confirmation of the results presented in Table 1, we have performed absolute phase noise measurements of the laser beatnote, shown in Fig. 7, in two different situations. In the first one, only the DFB laser is locked while the ECDL is free-running. In this case, the phase noise spectrum exhibits a clear $f^{-3}$ slope, signature of a flicker frequency noise. Following the well-known power law such that $S_{\varphi}(f)=\sum_{i=-4}^{0} b_{i} f^{i}$ [47], we extract $b_{-3}=93 \mathrm{dBrad}^{2} / \mathrm{Hz}$, yielding an expected Allan deviation $\sigma_{y}(1 s)=\sqrt{2 \ln 2 \frac{b_{-3}}{v_{0}^{2}}}=1.6 \times 10^{-10}$. When both lasers are locked, the slope of the phase noise spectrum turns to $f^{-2}$, signature of the expected white frequency noise, for offset frequencies lower than about $1.5 \mathrm{kHz}$. In this case, we extract $b_{-2}=53 \mathrm{dBrad}^{2} / \mathrm{Hz}$, yielding an expected Allan deviation $\sigma_{y}(1 s)=\sqrt{\frac{b_{-2}}{v_{0}^{2}}}=9.4 \times 10^{-13}$. This value is in agreement with the expected $1.6 \times 10^{-12}$ stability limit reported in Table 1 from the detection noise budget.

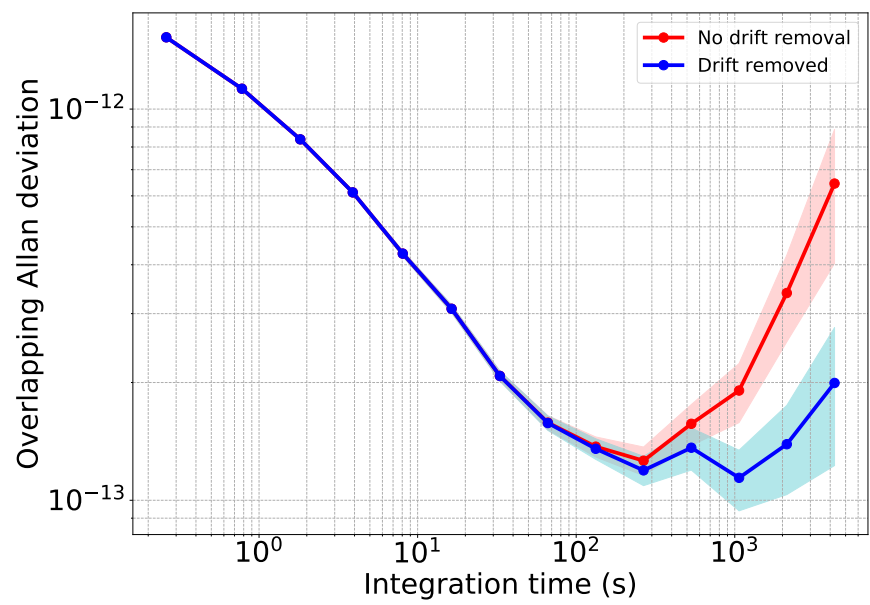

Fig. 8. Overlapping Allan deviation of the laser beatnote, with or without drift removed.

\section{Short-term frequency stability}

We performed a measurement of the laser beatnote short-term fractional frequency stability. In this test, the laser power is about $450 \mu \mathrm{W}$ at the input of both cells and cell temperatures are fixed at 62 and $63^{\circ} \mathrm{C}$, for the ECDL and DFB, respectively. Results are shown on Fig. 8.

The Allan deviation is $1.1 \times 10^{-12} \tau^{-1 / 2}$ for integration times up to $100 \mathrm{~s}$. This result is in good agreement with the short-term stability expected from the detection noise budget $\left(1.6 \times 10^{-12}\right)$ and absolute phase noise performances $\left(9.8 \times 10^{-13}\right)$, reported in section $B$. These stability results are competitive with those obtained in [21,22] (DFB laser-based Rb microcell optical frequency reference) for integration times up to $100 \mathrm{~s}$ and are encouraging for further exploration of this approach. They also compare favorably with recent results reported with DFSDS in a $10 \mathrm{~cm}$ long $\mathrm{Rb}$ vapor cell [48]. As suggested by our detection noise budget, the individual stability of the DFSDS-based microcell ECDL should be at the level of $8 \times 10^{-13}$ at $1 \mathrm{~s}$ in current conditions and should even be further improved by increasing the laser modulation frequency and servo bandwidth to a few hundreds of $\mathrm{kHz}$. These previsions will be experimentally tested in a near future by comparing the present microcell-stabilized ECDL to an ultra-stable $895 \mathrm{~nm}$ signal generated from a lab-prototype cavity-stabilized laser [49], with the help of an optical frequency comb.

For integration times higher than $100 \mathrm{~s}$, we currently observe a degradation of the clock stability, yielding the level of $1.9 \times$ $10^{-13}$ at $10^{3} \mathrm{~s}$ and $6.3 \times 10^{-13}$ at $4000 \mathrm{~s}$. We note that this result at $4 \times 10^{3} \mathrm{~s}$ is more than 10 times better than the one reported in our previous study [42]. This improvement is probably due to an improved global thermal control of the experiment. The blue curve in Fig. 8 shows the deviation with application of a linear drift removal on experimental frequency data. In this case, the Allan deviation is slightly improved for $\tau>250 \mathrm{~s}$, yielding $2 \times 10^{-13}$ at $4 \times 10^{3} \mathrm{~s}$. Studies are in progress to evaluate the contribution of main frequency shifts and improve the lasers' mid-term stabilities.

\section{CONCLUSIONS}

In conclusion, we have characterized the short-term stability budget of two lasers (one DFB laser and one ECDL) frequencystabilized with Cs microfabricated cells using the dual-frequency 
sub-Doppler spectroscopy (DFSDS) technique. The laser beatnote short-term frequency stability was measured at the level of $1.1 \times 10^{-12}$ at $1 \mathrm{~s}$, in good agreement with the detection noise budget and absolute phase noise measurements. The short-term stability of the laser beatnote is limited by the intermodulation effect contribution induced by the frequency noise of the DFB laser. We believe that demonstrating a frequency stability at $1 \mathrm{~s}$ in the $10^{-13}$ range, using a low noise ECDL locked to a microfabricated cell with a high servo bandwidth (a few $100 \mathrm{kHz}$ ), is a reasonable objective with the DFSDS approach. Further studies will be performed in a near future in this direction, combined with efforts to improve the laser mid-term stability, currently at the level of $6.3 \times 10^{-13}$ at $4 \times 10^{3} \mathrm{~s}$. In addition, the enhanced compacity of the DFSDS-based laser system might be envisioned by using a directly-modulated laser [50] (to suppress the EOM), by replacing the AOM with a liquid crystal device or by benefiting from integrated photonics systems [51, 52].

\section{FUNDING}

This work was supported in part by Centre National des Etudes Spatiales (CNES) through the OSCAR project (grant 200837/00), in part by Région de Franche-Comté with NOUGECELLL project (grant 20174-06245), and in part by Agence Nationale de la Recherche (ANR) in the frame of the LabeX FIRST-TF (Grant ANR 10-LABX-0048), EquipX Oscillator-IMP (Grant ANR 11EQPX-0033) projects. The authors also thank the microfabrication platform MIMENTO and the Graduate School EIPHI from Université Bourgogne-Franche Comté. D. Brazhnikov was supported by Russian Science Foundation (grant 17-72-20089).

\section{ACKNOWLEDGMENT}

The authors thank Philippe Abbé and Gilles Martin (FEMTO-ST) for help with the development of the microcell physics package and electronics. The authors acknowledge F.-X. Esnault and J. Delporte (CNES) for their expertise and sharp appreciation of this study.

\section{DISCLOSURES}

The authors declare no conflicts of interest.

\section{DATA AVAILABILITY}

Data underlying the results presented in this paper are not publicly available at this time but may be obtained from the authors upon reasonable request.

\section{REFERENCES}

1. J. Kitching, Chip-scale atomic devices, Appl. Phys. Rev. 5, 031302 (2018).

2. J. Vanier, Atomic clocks based on coherent population trapping: a review, Appl. Phys. B 8, 4, 421-442 (2005).

3. https://www.microsemi.com/product-directory/clocksfrequency-references/3824-chip-scale-atomic-clock-csac

4. R. Lutwak, A. Rahsed, M. Varghese, G. Tepolt, J. LeBlanc, M. Mescher, D. K. Serkland, K. M. Geib, G. M. Peake and S. Romisch, The Chip-Scale Atomic Clock-Prototype Evaluation, Proceedings of the $39^{\text {th }}$ Annual Precise Time and Time Interval Meeting, pp. 269-290 (2007).

5. H. Zhang, H. Hans, A. Tharayil Narayanan, A. Shirane, M. Suzuki, K. Arasaka, K. Adachi, S. Goka, S. Yanagimachi, K. Okada, ULPAC: A Miniaturized ultralow-power atomic clock, IEEE Journ. Solid State Circ. 54, 11, 3135-3148 (2019).
6. R. Vicarini, M. Abdel Hafiz, V. Maurice, N. Passilly, E. Kroemer, L. Ribetto, V. Gaff, C. Gorecki, S. Galliou and R. Boudot, Mitigation of temperature-induced light-shift effects in miniaturized atomic clocks, IEEE Ultrason. Ferroelec. Freq. Contr. 66, 12, 1962-1967 (2019).

7. S. Yanagimachi, K. Harasaka, R. Suzuki, M. Suzuki and S. Goka, Reducing frequency drift caused by light shift in coherent population trapping-based low-power atomic clocks, Appl. Phys. Lett. 116, 104102 (2020).

8. Y. Zhang and W. Yang and S. Zhang and J. Zhao, Rubidium chipscale atomic clock with improved long-term stability through light intensity optimization and compensation for laser frequency detuning, J. Opt. Soc. Am. B 33, 8, 1756-1763 (2016).

9. O. Kozlova, S. Guérandel and E. de Clercq, Temperature and pressure shift of the Cs clock transition in the presence of buffer gases: $\mathrm{Ne}, \mathrm{N}_{2}$, Ar, Phys. Rev. A 83, 062714 (2011).

10. J. Guéna, M. Abgrall, D. Rovera, P. Laurent, B. Chupin, M. Lours, G. Santarelli, P. Rosenbusch, M. Tobar, R. Li, K. Gibble, A. Clairon and S. Bize, Progress in atomic fountains at LNE-SYRTE, IEEE Trans. Ultrason. Ferroelec. Freq. Contr. 59, 3, 391-410 (2012).

11. M. Schioppo, R. C. Brown, W. F. McGrew, N. Hinkley, R. J. Fasano, K. Beloy, T. H. Yoon, G. Milani, D. Nicolodi, J. A. Sherman, N. B. Phillips, C. W. Oates and A. Ludlow, Ultrastable optical clock with two cold-atom ensembles, Nat. Photon. 11, 48 (2017).

12. J. Grotti, S. Koller, S. Vogt, S. Hafner, U. Sterr, C. Lisdat, H. Denker, C. Voigt, L. Timmen, A. Rolland, F. N. Baynes, H. S. Margolis, M. Zampaolo, P. Thoumany, M. Pizzocaro, B. Rauf, F. Bregolin, A. Tampellini, P. Barbieri, M. Zucco, G. A. Costanzo, C. Clivati, F. Levi and D. Calonico, Geodesy and metrology with a transportable optical clock, Nature Physics 14, 437 (2018).

13. W. F. McGrew, X. Zhang, R. J. Fasano, S. A. Schaffer, K. Beloy, D. Nicolodi, R. C. Brown, N. Hinkley, G. Milani, M. Schioppo, T. H. Yoon and A. Ludlow, Atomic clock performance enabling geodesy below the centimetre level, Nature 564, 87-90 (2018).

14. P. Wcislo, P. Ablewski, K. Beloy, S. Bilicki, M. Bober, R. Brown, R. Fasano, R. Cyurilo, H. Hachisu, T. Ido, J. Lodewyck, A. Ludlow, W. McGrew, P. Morzynski, D. Nicolodi, M. Schioppo, M. Sekido, R. Le Targat, P. Wolf, X. Zhang, B. Zjawin and M. Zawada, New bounds on dark matter coupling from a global network of optical atomic clocks, Science Advances 4, 12 (2018).

15. C. Sanner, N. Huntemann, R. Lange, C. Tamm, E. Peik, M. Safranova and S. G. Porsev, Optical clock comparison for Lorentz symmetry testing, Nature 567, 204-208 (2019).

16. E. Oelker, R. B. Hutson, C. J. Kennedy, L. Sonderhouse, T. Bothwell, A. Goban, D. Kedar, C. Sanner, J. M. Robinson, G. E. Marti, D. G. Matei, T. Legero, M. Giunta, R. Holzwarth, F. Riehle, U. Sterr and J. Ye, Demonstration of $4.8 \times 10^{-17}$ stability at $1 \mathrm{~s}$ for two independent optical clocks, Nature Photonics 13, 714-719 (2019).

17. T. Schuldt, M. Gohlke, M. Oswald, J. Wüst, T. Blomberg, K. Döringshoff, A. Bawamia, A. Wicht, M. Lezius, K. Voss, M. Krutzik, S. Hermann, E. Kovalchuk, A. Peters and C. Braxmaier, Optical clock technologies for global navigation satellite systems, GPS Solutions 25, 83 (2021).

18. D. Touahri, O. Acef, A. Clairon, J. J. Zondy, R. Felder, L. Hilico, B. de Beauvoir, F. Biraben and F. Nez, Frequency measurement of the 5S1/2 (F=3)-5D5/2 (F=5) two-photon transition in rubidium, Optics communications 133, 471-478 (1997).

19. K. W. Martin, G. Phelps, N. D. Lemke, M. S. Bigelow, B. Stuhl, M. Wojcik, M. Holt, I. Coddington, M. W. Bishop, and J. H. Burke, Compact optical atomic clock based on a two-photon transition in rubidium, Phys. Rev. Applied 9, 014019 (2018).

20. K. W. Martin, B. Stuhl, J. Eugenio, M. S. Safronova, G. Phelps, J. H. Burke, and N. D. Lemke., Frequency shifts due to Stark effects on a rubidium two-photon transition, Phys. Rev. A. 100, 023417 (2018).

21. Z. L. Newman, V. Maurice, T. Drake, J. R. Stone, T. C. Briles, D. T. Spencer, C. Fredrick, Q. Li, D. Westly, B. R. Ilic, B. Shen, M-G. Suh, K. Y. Yang, C. Johnson, D. M. S. Johnson, L. Hollberg, K. J. Vahala, K. Srinivasan, S. A. Diddams, J. Kitching, S. B. Papp and M. 
T. Hummon, Architecture for the photonic integration of an optical atomic clock, Optica 6, 5, 680 (2019).

22. V. Maurice, Z. L. Newman, S. Dickerson, M. Rivers, J. Hsiao, P. Greene, M. Mescher, J. Kitching, M. T. Hummon and C. Johnson, Miniaturized optical frequency reference for next-generation portable optical clocks, Opt. Exp. 28, 17, 24708 (2020).

23. Z. L. Newman, V. Maurice, C. Fredrick, T. Fortier, H. Leopardi, L. Hollberg, S. Diddams, J. Kitching and M. T. Hummon, Highperformance compact optical standard, ArXiv 2105.00610 (2021).

24. V. Gerginov and K. Beloy, Two-photon optical frequency reference with active ac Stark shift cancellation, Phys. Rev. Appl. 10, 014031 (2018).

25. C. Perrella, P. S. Light, J. D. Anstie, F. N. Baynes, R. T. White, and A. N. Luiten, Dichroic two-photon rubidium frequency standard, Phys. Rev. Appl. 12, 054063 (2019).

26. S. Haroche and F. Hartmann, Theory of saturated-absorption line shapes, Phys. Rev. A 6, 1280 (1972)

27. C. Wieman and T. W. Hänsch, Doppler-free laser polarization spectroscopy, Phys. Rev. Lett. 36, 1170 (1976).

28. G. D. Rovera, G. Santarelli and A. Clairon, A laser diode system stabilized on the cesium $\mathrm{D}_{2}$ line, Rev. Sci. Instr. 65, 5, 1502 (1994).

29. C. Affolderbach and G. Mileti, Tuneable, stabilised diode lasers for compact atomic frequency standards and precision wavelength references, Optics and Lasers in Engineering 43, 291-302 (2005).

30. X. Liu and R. Boudot, A Distributed-feedback diode laser frequency stabilized on Doppler-Free Cs $D_{1}$ line, IEEE Trans. Instr. Meas. 61, 10, 2852 (2011).

31. W. Liang, V. S. Ilchenko, D. Eliyahu, E. Dale, A. A. Savchenkov, D. Seidel, A. B. Matsko and L. Maleki, Compact stabilized semiconductor laser for frequency metrology, Appl. Opt. 54, 11, 3353 (2015).

32. W. Liang, V. S. Ilchenko, D. Eliyahu, A. A. Savchenkov, A. B. Matsko, D. Seidel and L. Maleki, Ultralow noise miniature external cavity semiconductor laser, Nature Comm. 6, 7371 (2015).

33. A. Strangfeld, S. Kanthak, M. Schiemangk, B. Wiegand, A. Wicht, A. Ling and M. Krutzik, Prototype of a compact rubidium-based optical frequency reference for operation on nanosatellites, J. Opt. Soc. Am. B 38, 6, 1885-1890 (2021).

34. S. Knappe, H. G. Robinson and L. Hollberg, Microfabricated saturated absorption laser spectrometer, Optics Express 15, 10, 6293 (2007)

35. F. Gruet, F. Vecchio, C. Affolderbach, F. Pétremand, N. F. de Rooij, T. Maeder and G. Mileti, A miniature frequency stabilized VCSEL system emitting at $795 \mathrm{~nm}$ based on LTCC modules, Opt. Lasers Eng. 51, 8, 1023-1027 (2013).

36. W. Loh, M. T. Hummon, H. F. Leopardi, T. M. Fortier, F. Quinlan, J. Kitching, S. B. Papp and S. A. Diddams, Microresonator Brillouin laser stabilization using a microfabricated rubidium cell, Opt. Exp. 24, 13, 14516 (2016).

37. M. T. Hummon, S. Kang, D. G. Bopp, Q. Li, D. A. Westly, S. Kim, C. Fredrick, S. A. Diddams, K. Srinivasan, V. Aksyuk and J. E. Kitching, Photonic chip for laser stabilization to an atomic vapor with $10^{-11}$ instability, Optica 5, 4, 443 (2018).

38. L. Stern, J. R. Stone, S. Kang, D. C. Cole, M. G. Suh, C. Fredrick, Z. Newman, K. Vahala, J. Kitching, S. A. Diddams and S. B. Papp, Direct Kerr frequency comb atomic spectroscopy and stabilization, Science Advances 6, eaax6230 (2020).

39. W. Zhang, L. Stern, D. Carlson, D. Bopp, Z. Newman, S. Kang, J. Kitching and S. B. Papp, Ultranarrow linewidth photonic-atomic laser, Laser and Photonics Reviews (2020). https://doi.org/10.1002/lpor.201900293

40. M. Abdel Hafiz, G. Coget, E. de Clercq and R. Boudot, Doppler-free spectroscopy on the Cs D1 line with a dual-frequency laser, Opt. Lett. 41, 2982 (2016).

41. M. Abdel Hafiz, D. Brazhnikov, G. Coget, A. Taichenachev, V. Yudin, E. de Clercq and R. Boudot, High-contrast sub-Doppler absorption spikes in a hot atomic vapor cell exposed to a dual-frequency laser field, New Journ. Phys. 19, 073028 (2017).
42. D. Brazhnikov, M. Petersen, G. Coget, N. Passilly, V. Maurice, C. Gorecki and R. Boudot, Dual-frequency sub-Doppler spectroscopy: Extended theoretical model and microcell-based experiments, Phys. Rev. A 99, 062508 (2019).

43. F. Tricot, D. H. Phung, M. Lours, S. Guérandel and E. de Clercq, Power stabilization of a diode laser with an acousto-optical modulator, Rev. Sci. Instr. 89, 113112 (2018).

44. R. Vicarini, V. Maurice, M. Abdel Hafiz, J. Rutkowski, C. Gorecki, N. Passilly, L. Ribetto, V. Gaff, V. Volant, S. Galliou and R. Boudot, Demonstration of the mass-producible feature of a Cs vapor microcell technology for miniature atomic clocks, Sens. Act. Phys. A 280, 99-106 (2018).

45. P. Yun, F. Tricot, C. E. Calosso, S. Micalizio, B. Francois, R. Boudot, S. Guérandel and E. de Clercq, High-Performance coherent population trapping clock with polarization modulation, Phys. Rev. Appl. 7, 014018 (2017).

46. C. Audoin, V. Candelier and N. Dimarcq, A limit to the frequency stability of passive frequency standards due to an intermodulation effect, IEEE Trans. Instr. Meas. 40, 2, 121-125 (1991).

47. E. Rubiola, Phase noise and frequency stability in oscillators, Cambridge University Press, ISBN 9780521153287 (2012).

48. M. Zhao, X. Jiang, R. Fang, Y. Qiu, Z. Ma, C. Han, B. Lu and C. Lee, Laser frequency stabilization via bichromatic Doppler-free spectroscopy of an ${ }^{87} \mathrm{Rb}$ D1 line, Appl. Opt. 60, 17, 5203-5207 (2021).

49. A. Didier, J. Millo, S. Grop, B. Dubois, E. Bigler, E. Rubiola, C. Lacroûte, Y. Kersalé, Ultra-low phase noise all-optical microwave generation setup based on commercial devices, Appl. Opt. 54, 12, 3682-3685 (2015).

50. P. Yun, Q. Li, Q. Hao, G. Liu, E. de Clercq, S. Guérandel, X. Liu, S. $\mathrm{Gu}, \mathrm{Y}$. Gao and S. Zhang, High-performance coherent population trapping atomic clock with direct-modulation distributed Bragg reflector laser, ArXiv 2103:00707 (2021).

51. C. Wang, M. Zhang, B. Stern, M. Lipson and M. Loncar, Nanophotonic lithium niobate electro-optic modulators, Opt. Exp. 26, 2, 1547 (2019).

52. C. J. Sarabalis, T. P. McKenna, R. N. Patel, R. Van Laer and A. H. Safavi-Naeini, Acousto-optic modulation in lithium niobate on sapphire, APL Photonics 5, 086104 (2021). 